\title{
MicroRNA therapeutics - back in vogue?
}

Abbreviations: miRNAs, microRNAs; RNAi, RNA interference; dsRNA, double-stranded RNA; MPM, malignant pleural mesothelioma; NSCLC, non-small cell lung cancer

\section{Editorial}

MicroRNAs (miRNAs) are a class of small non-coding RNAs involved in post-transcriptional control of gene expression. They form a complex network where each miRNA regulates multiple mRNAs and each mRNA is regulated by multiple miRNAs. MicroRNAs seem to impinge on almost every cellular process and the dysregulation of miRNA activity is associated with disease. ${ }^{1,2}$ Being both small in size and having essential functions in many diseases, miRNAs present attractive targets for therapeutic development. For more than a decade, in the development of commercial RNA interference (RNAi) therapeutics, one word best describes this space - volatility. As early 2002, many biotechnology companies focused on the therapeutic development of RNAi began appearing, including Ribozyme Pharmaceuticals (Sirna Therapeutics), Atugen AG (Silence Therapeutics), Mirna Therapeutics, Provita (Tekmira), Regulus (Alnylam Pharmaceuticals and ISIS Pharmaceuticals) and Quark Pharmaceuticals. Following the global financial crisis in 2008, we have seen many major pharmaceutical companies like Pfizer, Merck/ Allergan, Medtronic, Novartis, Abbott Labs and Roche exit the RNAi therapeutics development (problems with expired patents and in vivo specific delivery chemistries aside).

Nevertheless, recent events such as Roche's acquisition of Santaris Pharma A/S, Regulus and Sanofi's RG-012 preclinical model of Alport Syndrome, and Miravirsen's clinical testing of SPC3649 for treatment of hepatitis $\mathrm{C}$ virus infections, to name a few, have seen an reinvigoration in the field of RNAi therapeutics. This continues the momentum that followed the award of the Nobel Prize in Medicine to Andrew $\mathrm{Z}$ Fire and Craig $\mathrm{C}$ Mello for their role in the discovery of RNAi through double-stranded RNA (dsRNA). Add to this the robust scientific rationales and an improvement in delivery approaches and anti-immunostimulatory strategies, the therapeutic RNAi skeptics are slowly becoming convinced. With the global investment for RNAi therapeutics improving, the picture is once again rosy. ${ }^{3}$ In the cancer field, RNAi therapeutics have been proposed as a means to inhibit otherwise undruggable targets, but they still suffer from the 'one drug, one target' problem that affects traditional cancer drug development. MicroRNAs, via their ability to control multiple mRNA targets in distinct pathways, have the potential to overcome this limitation. With their central role in tumor biology and global downregulation in many cancers, they make attractive therapeutic targets. Learning from the experience of those in the RNAi field, miRNA replacement as a therapeutic approach has predominantly made use of synthetic miRNA mimics, similar in structure to small interfering RNAs, to restore lost tumor suppressor activity. ${ }^{4}$ Restoration of lost tumor suppressor miRNAs using synthetic dsRNAs (with a delivery agent) has been successful in several models. These include: systemic delivery of miR$34 \mathrm{a}$ and let-7 mimics to lung xenograft tumors using a neutral lipid emulsion; 5,6 intratumoral injection of miR-143 mimics to colorectal tumors using cationic lipid ${ }^{7}$ and delivery of miR-16 mimic to bone metastases in a prostate cancer model using atelocollagen. ${ }^{8}$ Recently

\author{
Volume I Issue 2 - 2014
}

\author{
Ruby CY Lin, ${ }^{1,2}$ Nico van Zandwijk, ${ }^{1,3}$ Glen \\ Reid ${ }^{1,3}$ \\ 'Asbestos Diseases Research Institute, Australia \\ ${ }^{2} \mathrm{~S} c h o o l$ of Biotechnology and Biomolecular Sciences, University \\ of New South Wales, Australia \\ ${ }^{3}$ School of Medicine, University of Sydney, Australia
}

\begin{abstract}
Correspondence: Ruby CY Lin,Asbestos Diseases Research Institute, Bernie Banton Centre, Gate 3, Hospital Road, Concord Hospital, NSW 2139, Australia, Tel +6I 29767 9807,
\end{abstract}

Email ruby.lin@sydney.edu.au

Received: November 21, 2014 | Published: November 22, 2014

the miR-34a mimic (MRX34, Mirna Therapeutics) encapsulated using an innovative liposomal formulation called SMARTICLES ${ }^{\circ}$ entered Phase I clinical trial in patients with hepatocellular carcinoma. This is notable in being the first microRNA mimic to be trialed in patients, and also because it targets the liver, traditionally the only organ that receives pharmacologically relevant concentration of drug following systemic delivery of lipid-complexed siRNA.

The targeted delivery of microRNA mimics administered systemically remains a challenge in the field. Several delivery vehicles now exist with the potential to overcome this hurdle. In the thoracic cancer landscape, we are currently running a Phase I clinical trial in three major hospitals in Sydney, Australia, treating malignant pleural mesothelioma (MPM) and non-small cell lung cancer (NSCLC) patients with "TargomiRs", an experimental therapy consisting of synthetic miR-16-based microRNA mimics encapsulated in $\mathrm{EDV}^{\mathrm{TM}}$ nanocells, ${ }^{9}$ and targeted with Epidermal Growth Factor Receptor-specific antibodies. Similar to previous findings in NSCLC, we found that MPM is characterized by downregulation of the miR-15/16 family, and restoring expression leads to tumor growth inhibition and induction of apoptosis both in vitro and in vivo. ${ }^{2}$ Our TargomiR approach is unique in comparison to other miRNA-based approaches, in that it aims to correct defects in the expression of an entire miRNA family, thereby restoring control over numerous dysregulated pathways common to both thoracic cancers. Both the sequences (TargomiRs) and $\mathrm{EDV}^{\mathrm{TM}}$ nanocells are patent protected and the use of cytotoxic drug- or nucleic acid-containing EDV ${ }^{\mathrm{TM}}$ nanocells has passed proof-of-concept stage in animal models ( $>3000$ nude mice, 37 dogs and 90 monkeys), as well as 20 patients with no severe adverse events (EnGeneIC unpublished data).The combinatorial use of designer miRNA mimics and the unique delivery strategy using $\mathrm{EDV}^{\mathrm{TM}}$ nanocells results in $\sim 30 \%$ of the administered dose reaching the tumor. Most importantly, there are no other vehicles on the current market that achieve the same level of delivery. This is the first of its kind worldwide for thoracic cancers, and just the second to use microRNA replacement as a therapeutic strategy.

The shift towards a renewed interest of RNAi therapeutics in general, and miRNA therapeutics in particular, by pharmaceutical companies creates economic opportunities for industries, companies 
and governments. The emerging market value for our TargomiRs, for example is primarily shaped by the number of patients diagnosed with advanced NSCLC in 2012, i.e.11,280 in Australia and 1.61million cases worldwide $\left(12.7 \%\right.$ of the total cancer incidence) ${ }^{10}$ With an incidence of $>600$ new cases per year in Australia and an estimated global incidence of 40,000 cases, ${ }^{11}$ MPM will also significantly contribute to the market value. It is also important to note that a large percentage of patients presenting with NSCLC and MPM will not favourably respond to standard therapy and become eligible for novel treatment options such as TargomiRs. Similarly, the potential market for MRX34 is dictated by increasing incidences of primary liver cancer and intrahepatic bile duct cancer worldwide. According to the American Cancer Society, there will be $\sim 33,190$ new cases $(24,600$ in men and 8,590 in women) diagnosed in the U. S. and $\sim 23,000$ people $(15,870$ men and 7,130 women) will die of these cancers in 2014. Furthermore, liver cancer is also a leading cause of cancer mortality worldwide, accounting for more than 600,000 deaths each year. Adding to the renewed interest in miRNA-based therapeutics, the hype surrounding Illumina's HiSeqX Ten breaking the $\$ 1000$ genome barrier for human whole-genome sequencing, provides opportunities to systematically interrogate the in vivo efficacy of miRNA therapeutics. Will this marriage add value to the development of miRNA therapeutics? Watch this space.

\section{Acknowledgements}

None.

\section{Conflict of interest}

Author declares that there is no conflict of interest.

\section{References}

1. Bernardo BC, Gao XM, Winbanks CE, et al. Therapeutic inhibition of the miR-34 family attenuates pathological cardiac remodeling and improves heart function. Proc Nat Acad Sci USA. 2012;109(43):17615-17620.
2. Reid G, Pel ME, Kirschner MB, et al. Restoring expression of miR-16: a novel approach to therapy for malignant pleural mesothelioma. Ann oncol. 2013;24(12):3128-3135.

3. Haussecker D. The Business of RNAi Therapeutics in. Mol Ther Nucleic Acids. 2012;1:e8.

4. Henry JC, Azevedo-Pouly AC, Schmittgen TD. MicroRNA replacement therapy for cancer. Pharm res. 2011;28(12):3030-3042.

5. Wiggins JF, Ruffino L, Kelnar K, et al. Development of a lung cancer therapeutic based on the tumor suppressor microRNA-34. Cancer Res. 2010;70(14):5923-5930.

6. Trang P, Wiggins JF, Daige CL, et al. Systemic delivery of tumor suppressor microRNA mimics using a neutral lipid emulsion inhibits lung tumors in mice. Mol Ther. 2011;19(6):1116-1122.

7. Akao Y, Nakagawa Y, Hirata I, et al. Role of anti-oncomirs miR143 and -145 in human colorectal tumors. Cancer Gene Ther. 2010;17(6):398-408.

8. Takeshita F, Patrawala L, Osaki M, et al. Systemic delivery of synthetic microRNA-16 inhibits the growth of metastatic prostate tumors via downregulation of multiple cell-cycle genes. Mol Ther 2010;18(1):181-187.

9. MacDiarmid JA, Brahmbhatt H. Minicells: versatile vectors for targeted drug or si/shRNA cancer therapy. Curr opin biotechnol. 2011;22(6):909-916.

10. Ferlay J, Shin HR, Bray F, et al. Estimates of worldwide burden of cancer in 2008: GLOBOCAN 2008. Int $J$ Cancer. 2010;127(12):2893-2917.

11. Stayner L, Welch LS, Lemen R. The worldwide pandemic of asbestosrelated diseases. Annu Rev Public Health. 2013;34:205-216. 